\title{
On the Rapid Hardening and Exhaustion of Twinning in Magnesium Alloy
}

\author{
P.D. Wu ${ }^{\mathrm{a}, *}$, X.Q. Guo ${ }^{\mathrm{b}}$, H. Qiao ${ }^{\mathrm{a}}$, S.R. Agnew ${ }^{\mathrm{c}}$, D.J. Lloyd ${ }^{\mathrm{d}}$, J.D. Embury ${ }^{\mathrm{e}}$ \\ ${ }^{a}$ Department of Mechanical Engineering, McMaster University \\ Hamilton, Ontario L8S 4L7, Canada \\ ${ }^{\mathrm{b}}$ State Key Laboratory for Geomechanics and Deep Underground Engineering \\ China University of Mining and Technology, Xuzhou, Jiangsu 221116, China \\ ${ }^{c}$ Department of Materials Science and Engineering, University of Virginia, Charlottesville, \\ Virginia, U.S.A. \\ ${ }^{\mathrm{d}}$ Aluminum Materials Consultants \\ 106 Nicholsons Point Road, Bath, Ontario K0H 1G0, Canada \\ ${ }^{\mathrm{e}}$ Department of Materials Science and Engineering, McMaster University \\ Hamilton, Ontario L8S 4L7, Canada
}

\begin{abstract}
We numerically study the rapid hardening and possible role of exhaustion of twinning in magnesium alloys under twinning dominated conditions, based on the large strain elastic visco-plastic self-consistent model. It is found that upon the exhaustion of the tensile twinning, further deformation requires the material to activate Prismatic and Pyramidal slips, which have relative high critical resolved shear stress (CRSS). However, the stress level at the exhaustion of the tensile twinning is not high enough to activate Prismatic and Pyramidal slips. Consequently, the imposed strain increments must mainly be accommodated by elastic deformations, and the strain hardening rate becomes a large fraction of the elastic modulus. Therefore, it can be concluded that the rapid hardening is a composite response more associated with elasticity of a large volume fraction of the material, than the dislocation-dislocation interactions normally held responsible for strain hardening of metals.
\end{abstract}

Keywords: Twinning; Hardening; Polycrystal plasticity modeling; Magnesium alloys

* Corresponding author. Tel.: +1 (905) 525-9140 Ext. 20092; Fax: +1 (905) 572-7944; E-mail: peidong@mcmaster.ca (P.D. Wu) 


\section{Introduction}

Magnesium (Mg) and its alloys are of a hexagonal close-packed (HCP) crystallographic structure with low symmetry and, consequently, exhibit high anisotropy in mechanical behavior. Plastic deformation in HCP materials is accommodated by both slip and twinning. Twinning as an important plastic deformation mechanism in HCP materials has been extensively studied (see e.g. Arul Kumar et al. [1]; Balik et al. [2]; Barnett et al. [3, 4]; Ghaffari Tari et al. [5]; Guo et al. [6]; Jin et al. [7]; Kabirian et al. [8]; Kurukuri et al. [9]; Lynch et al. [10]; Mathis et al. [11]; McClelland et al. [12]; Niezgoda et al. [13]; Shi et al. [14]; Stanford et al. [15]; Steglich et al. [16]; Wang and Choo [17]; Wen et al. [18]; Wu et al. [19]; Xin et al. [20]). In Mg alloys, the most commonly observed twinning mode is the $\{10 \overline{1} 2\}<10 \overline{1} 1>$ extension twinning. It is well known that conventionally rolled magnesium sheet exhibits a basal texture, in which the basal planes typically lie preferentially in the plane with its normal parallel to the normal direction (ND). When such a rolled $\mathrm{Mg}$ alloy sheet is under uniaxial compression along the rolling direction (RD) or the transverse direction (TD), plastic deformation is dominated by tensile twinning at small strains because the $c$-axes of many grains are in the direction of Poisson extension. It has been generally accepted that under twinning dominated conditions, the yielding and immediate post-yielding plasticity is governed largely by the twin nucleation, whereas plastic deformation at higher strains is governed by twin growth and dislocation slip. A region of particularly low work-hardening, or in some cases slight work-softening, can be clearly detected for the finer-grained samples (e.g., Ghaderi and Barnett [21]; Barnett et al. [22]). This Lüderslike plateau in the macroscopic stress strain curves implies that the freshly nucleated twins proceed without additional increase in the applied stress. In coarser grained samples, it has been observed by Barnett et al. [23] that there may be some strain hardening within the immediate 
post-yield regime, presumably due to an increasing difficulty in twin nucleation with straining. After such a plateau-type region of low hardening, a steep increase in flow stress or a rapid hardening is also clearly observed. Depending on the initial texture, the hardening rate after the plateau in the macroscopic stress strain curve can attend a maximum value of one third of the elastic shear modulus (see Knezevic et al. [24]; Oppedal et al. [25]). It is this regime of rapid hardening which is of interest in the present paper.

It has been generally thought that the tensile twinning contributes to strain hardening through: (1) crystallographic lattice reoriented to a harder orientation (Lou et al. [26]), (2) the Basinski effect, i.e. a glissile-to-sessile transformation of dislocations already present in the region experiencing the twinning shear transformation (Basinski et al. [27]), and (3) a HallPetch-like effect resulted from grain refinement due to tensile twinning itself. However, Knezevic et al. [24] concluded that, based on their experiments on a rolled AZ31 alloy, these three mechanisms are unlikely to be high enough to explain the experimentally convinced rapid hardening. Instead, they postulated that (4) the thin contraction twins are very effective in strain hardening of the alloy by restricting the slip length associated with Pyramidal slip, i.e. another Hall-Petch-like effect. They demonstrated that this assumption is able to explain the key experimental feathers.

It is here hypothesized that the rapid hardening effect is induced by (5) the exhaustion of twinning. It can be rationalized that the tensile twinning reorients the material in the twinned region into a hard orientation. Further plastic deformation in the material inside the twin has to activate Pyramidal slip, which has much higher critical resolved shear stress (CRSS) comparing to tensile twinning. In short, the proposed mechanism is a natural consequence of item (1) above. However, the details presented here have not been systematically presented elsewhere, and it will 
be shown that there are specific, temporal problems with each of the previously proposed explanations (2)-(4). Regarding item (2), additional evidence has recently been produced to indicate that the Basinski effect does occur (Wang and Agnew [28]), however, it can only be held indirectly responsible for the present effect, as we will discuss later in the paper. Regarding item (3) the microstructure is most refined by twinning at early stages (where the strain hardening rate is low). By the time the strain hardening rate is high, the twins have largely consumed the grains and the refinement is no longer significant. Regarding item (4), it is suggested that compression twinning is mainly significant at later stages in the deformation (c.f. Oppedal et al. [25]), where strain hardening is slowing and/or localization is underway.

The purpose of this work is therefore to numerically investigate the rapid hardening induced by hypothesis (5), the exhaustion of twinning in Mg alloys. All the simulations reported in this paper are based on the large strain Elastic-Viscoplastic Self-Consistent (EVPSC) model developed by Wang et al. [29]. The EVPSC model has been successfully used to study effects of basal texture (Wang et al. [30]), formability of AZ31 sheet (Wang et al. [31]), lattice strain evolution in Mg alloys and steel (Wang et al. [32, 33]; Lee et al. [34]) and large strain behaviour of $\mathrm{Zr}$ alloys (Qiao et al. [35]). Wang et al. [36, 37] also developed a new constitutive model of Twinning and De-Twinning (TDT) for HCP materials. The TDT model has been implemented into the EVPSC framework. The EVPSC-TDT model has been applied to study inelastic deformation in Mg alloys during unloading (Wang et al. [38]), the Swift effect under free-end torsion (Guo et al. [39]), and lattice strain evolution associated with twinning and detwinning under cyclic loadings (Wu et al. [40]; Qiao et al. [41]; Wang et al. [42]). 


\section{The EVPSC-TDT model}

The EVPSC model is a completely general elastic visco-plastic, fully anisotropic, selfconsistent polycrystal model, applicable to large strains and to any crystal summary. The TDT model is a new physics-based crystal plasticity model of twinning and detwinning for HCP materials. In this section, we very briefly recapitulate the EVPSC-TDT model, mainly for the purpose of definition and notation. For details we refer readers to Wang et al. [29, 36, 37].

The plastic deformation of a crystal is assumed to be due to crystallographic slip and twinning on crystallographic system $\left(\boldsymbol{s}^{\alpha}, \boldsymbol{n}^{\alpha}\right)$, with $\boldsymbol{s}^{\alpha}$ and $\boldsymbol{n}^{\alpha}$ being respectively the slip/twinning direction and the normal of the slip/twinning plane for system $\alpha$. For Mg alloys, we usually consider Basal $\langle a\rangle(\{0001\}<11 \overline{2} 0>)$, Prismatic $\langle a\rangle(\{10 \overline{1} 0\}<11 \overline{2} 0>)$ and Pyramidal $\langle c+a\rangle(\{\overline{1} \overline{1} 22\}<\overline{1} \overline{1} 23>$ slip systems, and the $\{10 \overline{1} 2\}<\overline{1} 011>$ extension twin system.

Following Asaro and Needleman [43], the grain level plastic strain rate consists of the shear rate $\dot{\gamma}^{\beta}$ on slip/twinning system $\alpha$ as

$$
\boldsymbol{d}^{p}=\sum_{\alpha} \dot{\gamma}^{\alpha} \boldsymbol{P}^{\alpha}
$$

where $\boldsymbol{P}^{\alpha}=\left(\boldsymbol{s}^{\alpha} \boldsymbol{n}^{\alpha}+\boldsymbol{n}^{\alpha} \boldsymbol{s}^{\alpha}\right) / 2$ is the Schmid tensor. For both slip and twinning, the resolved shear stress $\tau^{\alpha}=\boldsymbol{\sigma}: \boldsymbol{P}^{\alpha}$ is the driving force for shear rate $\dot{\gamma}^{\alpha}$. For slip,

$$
\dot{\gamma}^{\alpha}=\dot{\gamma}_{0}\left|\tau^{\alpha} / \tau_{c r}^{\alpha}\right|^{\frac{1}{m}} \operatorname{sgn}\left(\tau^{\alpha}\right)
$$

For twinning,

$$
\dot{\gamma}^{\alpha}=\left\{\begin{array}{cc}
\dot{\gamma}_{0}\left|\tau^{\alpha} / \tau_{c r}^{\alpha}\right|^{\frac{1}{m}} & \tau^{\alpha}>0 \\
0 & \tau^{\alpha} \leq 0
\end{array}\right.
$$


where $\dot{\gamma}_{0}$ is a reference shear rate, $\tau_{c r}^{\alpha}$ is the critical resolved shear stress (CRSS), and $m$ is the strain rate sensitivity. The key assumption of the TDT model is that a grain has four potential operations associated with twinning and detwinning (Wang et al. [36]). Operation A is twin nucleation and initiates a twin band or 'child'. Operation B is a propagation of the child into the parent. Operations A and B increase the twin volume fraction and thus correspond to twinning. Operation $\mathrm{C}$ is a propagation of the parent into the child. Operation D splits the twin band and decreases the twin volume fraction through re-twinning. Operations $\mathrm{C}$ and $\mathrm{D}$ decrease the twin volume fraction and thus correspond to detwinning. The TDT model treats new twin band (child) as a new grain.

For both slip and twinning, the evolution of CRSS, $\tau_{c r}^{\alpha}$, is given by:

$$
\dot{\tau}_{c r}^{\alpha}=\frac{d \hat{\tau}^{\alpha}}{d \Gamma} \sum_{\chi} h^{\alpha \chi}\left|\dot{\gamma}^{\chi}\right|
$$

where $\Gamma=\sum_{\beta} \int\left|\dot{\gamma}^{\alpha}\right| d t$ is the accumulated shear strain in the grain, and $h^{\alpha \chi}$ are the latent hardening coupling coefficients, which empirically account for the obstacles on system $\alpha$ associated with the activity of system $\chi \cdot \hat{\tau}^{\alpha}$ is the threshold stress and is characterized by:

$$
\hat{\tau}^{\alpha}=\tau_{0}^{\alpha}+\left(\tau_{1}^{\alpha}+h_{1}^{\alpha} \Gamma\right)\left(1-\exp \left(-\frac{h_{0}^{\alpha}}{\tau_{1}^{\alpha}} \Gamma\right)\right)
$$

Here, $\tau_{0}, h_{0}, h_{1}$, and $\tau_{0}+\tau_{1}$ are the initial CRSS, the initial hardening rate, the asymptotic hardening rate, and the back-extrapolated CRSS, respectively.

Because it is rare that a grain can be fully twinned, a threshold twin volume fraction is defined in the model to terminate twinning. Consequently, the TDT model introduces two statistical variables: accumulated twin fraction, $V^{a c c}$, and effective twinned fraction, $V^{\text {eff }}$. More specifically, $V^{a c c}$ and $V^{\text {eff }}$ are the weighted volume fraction of the twinned region and volume 
fraction of twin terminated grains, respectively. The threshold volume fraction, $V^{\text {th }}$, is defined as $V^{\text {th }}=\min \left(1.0, A_{1}+A_{2} \frac{V^{\text {eff }}}{V^{\text {acc }}}\right)$, where $A_{1}$ and $A_{2}$ are two material constants. Although it is not employed in the present work, a single parameter version of the TDT model has already been developed (Qiao et al. [44]). Incidentally, it is natural to ask, "Why would twinning become exhausted prior to completely consuming the grains?" Though a complete answer is beyond the scope of the present paper, since the results hinge so critically on this notion of twin exhaustion, it merits some comment. One hypothesis, which is presently under evaluation by some of the authors, is that the twin boundaries accumulate debris, in the context of sweeping the surrounding dislocated crystals (Wang and Agnew [28]). It is suggested that this accumulation of debris could render further twin boundary motion difficult.

Coupling the single crystal plasticity outlined above to the aggregate response is accomplished using self-consistent approaches based upon the Eshelby inclusion formalism (Eshelby [45]). Various self-consistent schemes have been proposed. Wang et al. [46] have evaluated several self-consistent approaches by studying the large strain behaviour of magnesium alloy AZ31B sheet under tension and compression along different directions. It has been demonstrated that, of the approaches examined, the Affine self-consistent scheme gives the best overall performance. Therefore, the EVPSC-TDT model the Affine self-consistent scheme is employed in the present study.

\section{Results and Discussion}

In the present paper, we consider rolled $\mathrm{Mg}$ alloy sheets under in-plane uniaxial tension and compression. In all the simulations, a strain rate, $D_{11}=\dot{\varepsilon}=5 \times 10^{-3} \mathrm{~s}^{-1}$, is prescribed in the loading direction, and the macroscopic stress components are enforced to be zero, except for the 
stress $\sigma_{11}=\sigma$ along the loading direction. In all the figures shown in the present paper, stress $\sigma$ and strain $\varepsilon$ are presented in their absolute values, and stress is in unit of MPa.

As mentioned previously, the present work focuses on the rapid hardening observed in Mg alloy sheets under in-plane compression. We consider two AZ31B sheets: one with very strong basal texture and the other with strong basal texture. In order to assess effects of initial texture on the rapid hardening, we also consider two extreme cases: one with ideal basal texture [i.e. a single crystal with Euler angles $\left.\left(30^{\circ}, 0^{\circ}, 0^{\circ}\right)\right]$ and a random texture. Fig. 1 shows the initial textures represented in terms of the $\{0002\}$ pole figure. The sheet with strong basal texture (texture 3 in Fig. 1) has been studied experimentally by Jain and Agnew [47] and numerically by Wang et al. [46] based on the EVPSC model with the Predominant Twin Reorientation (PTR) scheme proposed by Tomé et al. [48], while the sheet with very strong basal texture (texture 2 in Fig. 1) has been investigated experimentally by Wu et al. [49] and numerically by Wang et al. [37] based on the EVPSC-TDT model.

In all the simulations reported in the present paper, the reference slip/twinning rate, $\dot{\gamma}_{0}$, and the rate sensitivity, $m$, are prescribed to be the same for all slip/twinning systems: $\dot{\gamma}_{0}=0.001 \mathrm{~s}^{-1}$ and $m=0.05$, respectively. The room temperature elastic constants of magnesium single crystal $C_{11}=58.0, C_{12}=25.0, C_{13}=20.8, C_{33}=61.2$ and $C_{44}=16.6$ (units of GPa) are used. The set of CRSSs and hardening parameters for each plastic deformation mode are estimated by fitting numerical simulations of uniaxial tension and compression along the RD to the corresponding experimental flow curves for the sheet with very strong basal texture reported by Jain and Agnew [47]. The uniaxial tension and compression true stress and true strain curves along the RD are presented in Fig. 2. The macroscopic yield stresses for uniaxial compression and tension are around $100 \mathrm{MPa}$ and $165 \mathrm{MPa}$, respectively, showing the strong 
tension/compression asymmetry associated with twinning. The maximum stresses in tension and compression are $275 \mathrm{MPa}$ and $400 \mathrm{MPa}$, respectively. Under uniaxial compression, after a low stress plateau in the stress and strain curve, the $\mathrm{Mg}$ sheet hardens dramatically resulting in the characteristic S-shape of the compressive flow curve. It is clear that the EVPSC-TDT model fits the experimental curves quite well. Table 1 lists the values of the material parameters obtained from these simulations. These parameters are used in all of the subsequent simulations. It should be emphasised that the main purpose of this paper is to assess the influence of the twinning on the rapid hardening, and that the overall results and conclusions are not particularly dependent on the above values of the material parameters. It is also worth mentioning that we repeated the simulations by assuming isotropic elasticity with a Young's modulus of $E=45 \mathrm{GPa}$ and Poisson's ratio of $v=0.33$. It was found that the predicted flow curves based on the isotropic elasticity assumption were almost the same as those shown in the Fig. 2. This could be easily understood because $\mathrm{Mg}$ crystals exhibit a nearly isotropic elasticity. For more efficiently interpreting results, it is appropriate to apply the isotropic elasticity assumption to all the simulations reported in the present paper.

Figure 3 shows the predicted stress and strain curves of the four initial textures under uniaxial compression along the RD. The result for texture 3 and its comparison with the corresponding experimental result have been represented in Fig. 2. The most striking observation from Fig. 3 is that the maximum stress increases with increasing basal texture. The difference in the maximum stress between the various initial textures seems to relate to the difference in the rapid hardening between the initial textures. The stronger the basal texture, the more rapid the strain hardening. It is found that the rapid hardening starts at around a strain of 0.04 for all the 
initial textures considered. It should be noted that the shear strain of 0.1289 associated with tensile twinning and the value of $A_{1}=0.60$ correspond to a maximum axial strain of about 0.04 .

The effects of initial texture and tensile twinning on the rapid hardening are more clearly exhibited in Fig. 4, which shows the normalized hardening rate $\left(\frac{d \sigma / d \varepsilon}{E}\right)$ as a function of axial strain $\varepsilon$. It is found that in the case of ideal basal texture (texture 1) the work hardening rate can rapidly increase to a value close to $E$. The predicted maximum hardening rates, during the rapid hardening stage, are $0.4 E, 0.3 E$ and $0.1 E$ for the very strong basal texture (texture 2), strong basal texture (texture 3) and random texture (texture 4), respectively. For a comparison, the experimental hardening rate for the strong basal texture (texture 3 ) is also included. It is found that the EVPSC-TDT model accurately describes the rapid hardening. Fig. 4 also strongly suggests that the maximum hardening rate is proportional to the sharpness of the basal texture (see Fig. 1). On the other hand, it could be rationalized that the basal texture intensity will affect the fraction of orientations favoring the tensile twinning and thus eventually influences the hardening rate in the rapid hardening stage. The intensity is calculated as the ratio of the number of grains with a maximum orientational difference to the ideal basal orientation of less than $15^{0}$, to the total number of grains. The ideal grain orientation (texture 1) corresponds to perfect alignment of the basal pole with the ND. The calculated basal texture intensities are 5\%, 36\% and $68 \%$ respectively for texture 4 (random texture), texture 3 (strong basal texture) and texture 2 (very strong basal texture), while $100 \%$ for texture 1 (ideal basal orientation). The results shown in Fig. 4 imply that the maximum hardening rate is proportional to the basal intensity and there is a nonlinear relationship between the volume fraction of grains which are prone to undergo twinning and the maximum hardening rate. A question then arises: how initial texture and tensile twinning induce the rapid hardening shown in Figs. 3 and 4 ? 
Figure 5 presents relative activities of slip/twinning for the four initial textures under uniaxial compression along the RD. For the sheet with strong basal texture (texture 3), it is found that at strains $|\varepsilon|<0.04$, plasticity is accommodated by tensile twinning and Basal slip. When the tensile twining activity is dramatically reduced, at a strain $\varepsilon \approx-0.04$, the Basal slip and Prismatic slip activities significantly increase until $\varepsilon \approx-0.055$. After that, the activity of Prismatic slip decreases slowly with increasing strain, while the activities of Basal slip and Pyramidal slip increases steadily. For the random texture, Basal slip is the most important contributor, and the predicted activities in Prismatic slip and Pyramidal slip are low throughout the deformation process. The predicted activity of tensile twinning is significant and contributes about $17 \%$ of the plastic deformation at strains $|\varepsilon|<0.04$. After that, its activity decreases and it becomes essentially inactive at strains $|\varepsilon|>0.10$. For the ideal basal texture, the tensile twinning dominates the plastic deformation with very little activity of Basal slip at small strains. When the tensile twining activity dramatically reduces, at a strain $\varepsilon \approx-0.04$, the Basal slip activity rapidly increases and almost vertically jumps to nearly $100 \%$. Shortly after the peak, the EVPSC-TDT model predicts that the activity of Basal slip decreases rapidly to a very low level. Meanwhile, the Prismatic slip increases its activity dramatically to around $80 \%$ and then decreases quickly to about 50\%. During the rapid decrease of the activity in Prismatic slip, the activity of Pyramidal slip increases significantly up to about $42 \%$. At large strains, the single crystal deforms mainly through the Prismatic and Pyramidal slips with little contribution of Basal slip. The predicted activities for the sheet with very strong basal texture (texture 2) are found to be between those for the sheet with strong basal texture (texture 3) and the ideal basal texture (texture 1). The predicted activity levels have been validated by previous studies of internal strain (Wu et al. 
[40]) and texture evolution (Wang et al. [50]), during deformation of Mg alloy polycrystals with texture strengths similar to texture 2 or texture 3.

From Fig. 5, it becomes clear that at small strains the stronger the basal texture the more active the tensile twinning. Consequently, the twin volume fraction increases with increasing basal texture. At large strains, the activity of Basal slip decreases with increasing the basal texture. Dramatic changes in relative activities in slip/twinning are found at a strain $\varepsilon \approx-0.04$, where the tensile twinning is nearly exhausted. Notably, the EVPSC-TDT model predictions of twin volume fraction have been experimentally validated for a number of different $\mathrm{Mg}$ alloys and textures (Wang et al. [37, 50]; Wu et al. [19]).

It is believed by many that the rapid hardening is due to the exhaustion of twinning and the activation of the Pyramidal slip, since the CRSS of the latter is much higher than the basal slip and tensile twinning. However, comparing Fig. 3 with Fig. 4 shows that the Pyramidal slip is not activated until after the rapid hardening occurred. In other words, the strain interval from the moment of rapid hardening occurs to the activation of the Pyramidal slip is short but noticeable. On the other hand, it is noticed that during the rapid hardening the hardening rate can reach a value which approaches $E$, in the case of the ideal basal texture under uniaxial compression. Such a high hardening rate cannot be explained solely by the activation of Pyramidal slip. Rather, slip activation would lead to a lowering of the observed hardening rate. Thus, this extremely high hardening rate must, to some extent, be associated with elastic deformation. The necessary condition for verifying this hypothesis is that during the rapid hardening the plastic strain rate must be relatively small and the elastic strain rate is of a significant portion of the imposed/applied strain rate. 
Figure 6 shows the normalized macroscopic plastic strain rate $\frac{D_{11}^{P}}{D_{11}}$ (dashed lines), and normalized macroscopic elastic strain rate $\frac{D_{11}^{e}}{D_{11}}$ (solid lines) along the RD. Note that $D_{11}=\dot{\varepsilon}=5 \times 10^{-3} s^{-1}$ is the applied strain rate under uniaxial compression along the RD. At the initial elastic-plastic transition, the normalized elastic strain rate decreases and plastic strain rate increases rapidly. After the initial macroscopic yield stress and at strains $|\varepsilon|<0.04$, the imposed deformation is essentially accommodated by the plastic deformation and the elastic strain rate is very small. During the rapid hardening, the normalized elastic strain rate increases dramatically, while the plastic strain rate decreases quickly. For the ideal basal texture (texture 1), the normalized elastic strain rate approaches one. This implies that the deformation is nearly entirely elastic. The changes in the normalized elastic and plastic strain rates decrease with decreasing the basal texture. However, even in the sheet with a random texture (texture 4) the normalized elastic strain rate is appreciable. The predicted maximum normalized elastic strain rates are 0.4 , 0.3 and 0.1 for the very strong basal texture (texture 2), strong basal texture (texture 3 ) and random texture (texture 4), respectively. It is interesting to note that the trend of the predicted normalized hardening rate shown in Fig. 4 seems to follow the normalized elastic strain rate exhibited in Fig. 6.

Figure 7 shows the predicted normalized hardening rate $\frac{d \sigma / d \varepsilon}{E}$ (thin black solid lines) and normalized elastic strain rate $\frac{D_{11}^{e}}{D_{11}}$ (thick red dashed lines) as a function of the applied axial strain. It is clear that the $\left(\frac{d \sigma / d \varepsilon}{E} \sim \varepsilon\right)$ and $\left(\frac{D_{11}^{e}}{D_{11}} \sim \varepsilon\right)$ curves are almost on top each other for all 
the four initial textures considered. The relationship between the hardening rate and elastic strain rate can be easily understood by applying classical plasticity to analyze one-dimensional elasticplastic behaviour. Assume that $\sigma$ and $\varepsilon$ are the true stress and true strain along the loading direction, and that $\dot{\varepsilon}_{e}$ and $\dot{\varepsilon}_{p}$ are the elastic and plastic strain rate, respectively. The elastic constitutive model gives

$$
\dot{\sigma}=E \dot{\varepsilon}_{e}
$$

The hardening rate can be written as

$$
\frac{d \sigma}{d \varepsilon}=\frac{d \sigma}{d t} \frac{d t}{d \varepsilon}=\frac{\dot{\sigma}}{\dot{\varepsilon}}=\frac{E \dot{\varepsilon}_{e}}{\dot{\varepsilon}}
$$

Thus

$$
\frac{d \sigma / d \varepsilon}{E}=\frac{\dot{\varepsilon}_{e}}{\dot{\varepsilon}}
$$

The results shown in Fig. 7 strongly suggest that the rapid hardening is associated with the elastic straining.

To further verify the hypothesis that the rapid hardening is associated with elastic straining, Fig. 8 presents the predicted relative activities of slip/twinning modes in the matrix and twinned regions for the ideal basal texture. Note that the total relative activities were already shown in Fig. 5. The relative activity of twinning in the matrix is due to Operation A (the twin nucleation and formation of a twin band), while the relative activity of twinning in the twinned region is from Operation B (a propagation of the twin into the matrix). From Fig. 8, it is clear that, after the rapid hardening and at relatively large strains, the plastic deformation in the matrix is dominated by the Prismatic slip, which has the second highest CRSS (75 MPa) among all the plastic deformation modes considered. The plastic deformation in the twinned region is mainly accommodated by the Pyramidal slip, which has the highest CRSS (140 MPa), with a little help 
from the Basal slip. A careful observation indicates that the Prismatic slip is initiated in the matrix before the Pyramidal slip is activated in the twinned region (see Fig. 5).

From the numerical results presented above, it is observed that, for an $\mathrm{Mg}$ alloy sheet with a typical basal texture under in-plane uniaxial compression, the $c$-axes of a considerable number of grains must undergo extension. Therefore, tensile twinning makes a significant contribution to initial yielding and the plateau in the macroscopic stress strain curve immediate post-yielding. During this deformation stage, the easily activated Basal slip also accommodates plastic deformation. The relative activities of Prismatic and Pyramidal slips are almost zero or very small. Upon the exhaustion of the tensile twinning, further deformation of the sheet requires the activation of Prismatic and Pyramidal slip modes, which have relative high CRSS. However, the stress level at the exhaustion of the tensile twinning is not high enough to activate Prismatic and Pyramidal slips. Thus, the imposed strain increments must be accommodated by elastic deformations. In other words, after the exhaustion of the tensile twinning and before the activation of Prismatic and Pyramidal slips, the grain behaves like an elastic inclusion. The overall hardening of the polycrystal is proportional to the volume fraction of such elastic inclusions (see e.g. Taya et al. [51]).

Finally, it is worth mentioning that for a rolled magnesium sheet under cyclic loading (compression and then tension) along the $\mathrm{RD}$, tensile twinning and de-twinning are activated in the initial compression and subsequent tension, respectively. More specifically, the initial loading in compression activates twinning. During the compressive unloading and tensilereloading process detwinning dominates the response. Rapid hardening occurs again during the tensile straining, once the detwinning operation is complete (see e.g., Wu et al. [40] and Wang et al. [50]). Fig. 9 shows the measured and calculated stress and strain curves of an AZ31B sheet 
with very strong basal texture under cyclic loading along the RD (Fig. 9a), and the normalized hardening rate and elastic strain rate as functions of accumulated strain (Fig. 9b). The experimental data are taken from $\mathrm{Wu}$ et al. [40], and the values of the hardening parameters are from Guo et al. [39]. Both the first rapid hardening during the initial compression (upon the exhaustion of the tensile twinning) and the second rapid hardening regime which occurs during the tensile straining (once the detwinning operation is complete, i.e., exhausted) are associated with the elastic straining.

\section{Conclusions}

Using the EVPSC-TDT model, we have numerically studied the relationship between the exhaustion of twinning and the observed rapid hardening in magnesium alloys under twinning dominated conditions. It has been found that upon the exhaustion of the tensile twinning, further deformation of the material requires activation of Prismatic and Pyramidal slip modes, which have relative high CRSS. However, the stress level at the exhaustion of the tensile twinning is not high enough to activate Prismatic and Pyramidal slips. It has been numerically demonstrated that, in the strain (or time) interval from the exhaustion of twinning to the activation of Prismatic and Pyramidal slip modes, the imposed strain increments are mainly accommodated by elastic deformations. Since the rapid hardening occurs during the same interval, it has been concluded that the rapid hardening is associated with elasticity.

\section{Acknowledgement}

P.D. Wu and H. Qiao were supported by the Natural Sciences and Engineering Research Council of Canada (NSERC). X.Q. Guo was supported by the National Basic Research Program of China (2007CB209400) and the 111 Project of China (B07028). S.R. Agnew would like to thank the National Science Foundation of the U.S.A., grant number CMMI 1235259. 


\section{References}

[1] M. Arul Kumar, A.K. Kanjarla, S.R. Niezgoda, R.A. Lebensohn, C.N. Tomé, Numerical study of the stress state of a deformation twin in magnesium, Acta Materialia 84 (2015) 349-358.

[2] J. Balik, P. Dobron, F. Chmelik, R. Kuzel, D. Drozdenko, J. Bohlen, D. Letzig, P. Lukac, Modeling of the work hardening in magnesium alloy sheets, International Journal of Plasticity 76 (2015) 166-185.

[3] M.R. Barnett, O. Bouaziz, L.S. Toth, A microstructure based analytical model for tensile twinning in a rod textured Mg alloy, International Journal of Plasticity 72 (2015) 151 167.

[4] M.R. Barnett, A. Ghaderi, J. Quinta da Fonseca, J.D. Robson, Influence of orientation on twin nucleation and growth at low strains in a magnesium alloy, Acta Materialia 80 (2014) 380-391.

[5] D. Ghaffari Tari, M.J. Worswick, M.A. Gharghouri, Mechanical response of AZ31B magnesium alloy: Experimental characterization and material modeling considering proportional loading at room temperature, International Journal of Plasticity 55 (2014) 247-267.

[6] X.Q. Guo, A. Chapuis, P.D. Wu, S.R. Agnew, On twinning and anisotropy in rolled Mg alloy AZ31 under uniaxial compression, International Journal of Solids and Structures 64-65 (2015) 42-50.

[7] L. Jin, J. Dong, J. Sun, A.A. Luo, In-situ investigation on the microstructure evolution and plasticity of two magnesium alloys during three-point bending, International Journal of Plasticity 72 (2015) 218-232.

[8] F. Kabirian, A.S. Khan, T. Gnäupel-Herlod, Visco-plastic modeling of mechanical responses and texture evolution in extruded AZ31 magnesium alloy for various loading conditions, International Journal of Plasticity 68 (2015) 1-20.

[9] S. Kurukuri, M.J. Worswick, D. Ghaffari Tari, R.J. Mishra, J.T. Carter, Rate sensitivity and tension-compression asymmetry in AZ31B magnesium alloy sheet, Philosophical Transactions of The Royal Society A372 (2014) 20130216.

[10] P.A. Lynch, M. Kunz, N. Tamura, M.R. Barnett, Time and spatial resolution of slip and twinning in a grain embedded within a magnesium polycrystal, Acta Materialia 78 (2014) 203-212.

[11] K. Mathis, G. Csiszar, J. Capek, J. Gubicza, B. Clausen, P. Lukas, A. Vinogradov, S.R. Agnew, Effect of the loading mode on the evolution of the deformation mechanisms in randomly textured magnesium polycrystals - Comparison of experimental and modeling results, International Journal of Plasticity 72 (2015) 127-150.

[12] Z. McClelland, B. Li, S.J. Horstemeyer, S. Brauer, A.A. Adedoyin, L.G. Hector Jr, M.F. Horstemeyer, Geometrically necessary twins in bending of a magnesium alloy, Materials Science and Engineering A645 (2015) 298-305.

[13] S.R. Niezgoda, A.K. Kanjarla, I.J. Beyerlein, C.N. Tomé, Stochastic modeling of twin nucleation in polycrystals: An application in hexagonal close-packed metals, International Journal of Plasticity 56 (2014) 119-138.

[14] B. Shi, Y. Peng, F. Pan, A generalized thermodynamically consistent distortional hardening model for Mg alloys, International Journal of Plasticity 74 (2015) 158-174. 
[15] N. Stanford, R.K.W. Marceau, M.R. Barnett, The effect of high yttrium solute concentration on the twinning behaviour of magnesium alloys, Acta Materialia 82 (2015) 447-456.

[16] D. Steglich, X. Tian, J. Bohlen, T. Kuwabara, Mechanical testing of thin sheet magnesium alloys in biaxial tension and uniaxial compression, Experimental Mechanics 54 (2014) 1247-1258.

[17] Y. Wang, H. Choo, Influence of texture on Hall-Petch relationships in an Mg alloy, Acta Materialia 81 (2014) 83-97.

[18] W. Wen, M. Borodachenkova, C.N. Tome, G. Vincze, E.F. Rauch, F. Barlat, J.J. Gracio, Mechanical behavior of Mg subjected to strain path changes: Experiments and modeling, International Journal of Plasticity 73 (2015) 171-183.

[19] P.D. Wu, X.Q. Guo, H. Qiao, D.J. Lloyd, A constitutive model of twin nucleation, propagation and growth in Magnesium alloys, Materials Science and Engineering A625 (2015) 140-145.

[20] R. Xin, C. Ding, C. Guo, Q. Liu, Crystallographic analysis on the activation of multiple twins in rolled AZ31 Mg alloy sheets during uniaxial and plane strain compression, Materials Science and Engineering A652 (2015) 42-50.

[21] A. Ghaderi, M.R. Barnett, Sensitivity of deformation twinning to grain size in titanium and magnesium, Acta Materialia 59 (2011) 7824-7839.

[22] M.R. Barnett, M.D. Nave, A. Ghaderi, Yield point elongation due to twinning in a magnesium alloy, Acta Materialia 60 (2012) 1433-1443.

[23] M.R. Barnett, Z. Keshavarz, A.G. Beer, Influence of grain size on the compressive deformation of wrought Mg-3Al-1Zn. Acta Materialia 52 (2004), 5093-5103.

[24] M. Knezevic, A. Levinson, R. Harris, R.K. Mishra, R.D. Doherty, S.R. Kalidindi, Deformation twinning in AZ31: Influence on strain hardening and texture evolution, Acta Materialia 58 (2010) 6230-6242.

[25] A.L. Oppedal, H. El Kadiri, C.N. Tomé, G.C. Kaschner, S.C. Vogel, J.C. Baird, M.F. Horstemeyer, Effect of dislocation transmutation on modeling hardening mechanisms by twinning in magnesium, International Journal of Plasticity 30-31 (2012) 41-61.

[26] X.Y. Lou, M. Li, R.K. Boger, S.R. Agnew, R.H. Wagoner, Hardening evolution of AZ31B Mg sheet, International Journal of Plasticity 23 (2007) 44-86.

[27] Z.S. Basinski, M.S. Szczerba, M. Niewczas, J.D. Embury, S.J. Basinski, Transformation of slip dislocations during twinning of copper-aluminum alloy crystals, Revue de Metallurgie-Cahiers D Informations Techniques 94 (1997) 1037-1044.

[28] F. Wang, S.R. Agnew, Dislocation transmutation by tension twinning in magnesium alloy AZ31, International Journal of Plasticity 81 (2016) 63-86.

[29] H. Wang, P.D. Wu, C.N. Tomé, Y. Huang, A finite strain elastic-viscoplastic selfconsistent model for polycrystalline materials, Journal of the Mechanics and Physics of Solids 58 (2010) 594-612.

[30] H. Wang, P.D. Wu, M.A. Gharghouri, Effects of basal texture on mechanical behaviour of magnesium alloy AZ31B sheet, Materials Science and Engineering A527 (2010) 35883594.

[31] H. Wang, P.D. Wu, K.P. Boyle, K.W. Neale, On crystal plasticity formability analysis for magnesium alloy sheets, International Journal of Solids and Structures 48 (2011) 1000-1010. 
[32] H. Wang, P.D. Wu, C.N. Tomé, J. Wang, Study of lattice strains in magnesium alloy AZ31 based on a large strain elastic-viscoplastic self-consistent polycrystal model, International Journal of Solids and Structures 49 (2012) 2155-2167.

[33] H. Wang, B. Clausen, C.N. Tomé, P.D. Wu, Studying the effect of stress relaxation and creep on lattice strain evolution of stainless steel under tension, Acta Materialia 61 (2013) 1179-1188.

[34] S.Y. Lee, H. Wang, M.A. Gharghouri, G. Nayyeri, W. Woo, E. Shin, P.D. Wu, W.J. Poole, W. Wu, K. An, Deformation behavior of solid-solution-strengthened Mg-9wt\%Al alloy: In-situ neutron diffraction and elastic-viscoplastic self-consistent modeling, Acta Materialia 73 (2014) 139-148.

[35] H. Qiao, P.D. Wu, H. Wang, M.A. Gharghouri, M.R. Daymond, Evaluation of elasticviscoplastic self-consistent polycrystal plasticity models for zirconium alloys, International Journal of Solids and Structures 71 (2015) 308-322.

[36] H. Wang, P.D. Wu, C.N. Tomé, J. Wang, A constitutive model of twinning and detwinning for HCP polycrystals, Materials Science and Engineering A555 (2012) 93-98.

[37] H. Wang, P.D. Wu, J. Wang, C.N. Tomé, A physics-based crystal plasticity model for hexagonal close packed (HCP) crystals including both twinning and de-twinning mechanisms, International Journal of Plasticity 49 (2013) 36-52.

[38] H. Wang, P.D. Wu, J. Wang, Modeling inelastic behavior of magnesium alloys during cyclic loading-unloading, International Journal of Plasticity 47 (2013) 49-64.

[39] X.Q. Guo, W. Wu, P.D. Wu, H. Qiao, K. An, P.K. Liaw, On the Swift effect and twinning in a rolled magnesium alloy under free-end torsion, Scripta Materialia 69 (2013) 319-322.

[40] W. Wu, H. Qiao, K. An, X.Q. Guo, P.D. Wu, P.K. Liaw, Investigation of deformation dynamics in a wrought magnesium alloy, International Journal of Plasticity 62 (2014) $105-120$.

[41] H. Qiao, S.R. Agnew, P.D. Wu, Modeling twinning and detwinning behavior of $\mathrm{Mg}$ alloy ZK60A during monotonic and cyclic loading, International Journal of Plasticity 65 (2015) 61-84.

[42] H. Wang, S.Y. Lee, M.A. Gharghouri, P.D. Wu, S.G. Yoon, Deformation behavior of Mg-8.5wt.\%Al alloy under reverse loading investigated by in-situ neutron diffraction and elastic viscoplastic self-consistent modeling, Acta Materialia 107 (2016) 404-414.

[43] R.J. Asaro, A. Needleman, Texture development and strain hardening in rate dependent polycrystals, Acta Metall. 33 (1985) 923-953.

[44] H. Qiao, P.D. Wu, X.Q. Guo, S.R. Agnew, A new empirical equation for termination of twinning in magnesium alloys, Scripta Materialia 120 (2016) 71-75.

[45] J.D. Eshelby, The determination of the elastic field of an ellipsoidal inclusion, and related problems, Proceedings of the Royal Society of London A241 (1957) 376-396.

[46] H. Wang, B. Raeisinia, P.D. Wu, S.R. Agnew, C.N. Tomé, Evaluation of selfconsistent polycrystal plasticity models for magnesium alloy AZ31B sheet, International Journal of Solids and Structures 47 (2010) 2905-2917.

[47] A. Jain, S.R. Agnew, Modeling the temperature dependent effect of twinning on the behavior of magnesium alloy AZ31B sheet, Materials Science and Engineering A462 (2007) 29-36.

[48] C.N. Tomé, R.A. Lebensohn, U.F. Kocks, A model for texture development dominated by deformation twinning-application to zirconium alloys, Acta Metallurgica Et Materialia 39 (1991) 2667-2680. 
[49] L. Wu, S.R. Agnew, Y. Ren, D.W. Brown, B. Clausen, G.M. Stoica, H.R. Wenk, P.K. Liaw, The effects of texture and extension twinning on the low-cycle fatigue behavior of a rolled magnesium alloy, AZ31B, Materials Science and Engineering A527 (2010) 7057-7067.

[50] H. Wang, P.D. Wu, J. Wang, Numerical assessment of the role of slip and twinning in magnesium alloy AZ31B Sheet during loading path reversal, Metallurgical and Materials Transactions 46A (2015) 3079-3090.

[51] M. Taya, K.E. Levay, D.J. Lloyd, Strengthening of a particulate metal matrix composite by quenching, Acta Metallurgica Et Materialia 39 (1991) 73-87. 


\section{Figure Captions}

Figure 1. Initial textures presented in terms of the $\{0001\}$ pole figure. Textures $1-4$ are, from left to right, an ideal orientation with Euler angles $\left(30^{\circ}, 0^{\circ}, 0^{\circ}\right)$, a very strong basal texture of AZ31B from Wu et al. [49], a strong basal texture of AZ31B from Jain and Agnew [47] and a random texture.

Figure 2. Measured (symbols) and calculated (solid lines) stress and strain curves under uniaxial compression and tension along the RD. The initial texture (texture 3 in Fig. 1) and experimental stress and strain data are from Jain and Agnew [47].

Figure 3. Calculated effect of initial texture on stress and strain curve under uniaxial compression along the RD.

Figure 4. Calculated effect of initial texture on work hardening rate under uniaxial compression along the RD. The experimental data (open circles) for texture 3 are from Jain and Agnew [47].

Figure 5. Calculated effect of initial texture on relative activities of the slip modes and tensile twinning under uniaxial compression along the RD.

Figure 6. Calculated effect of initial texture on normalized plastic strain rate (dashed lines) and elastic strain rate (solid lines) under uniaxial compression along the RD.

Figure 7. Calculated normalized hardening rate (thin black solid lines) and normalized elastic strain rate (thick red dashed lines) under uniaxial compression along the RD.

Figure 8. Calculated relative activities of the slip modes and tensile twinning in the matrix and twins for the ideal basal texture. Note that the total of the relative activities were presented in Figure 5.

Figure 9. Measured and calculated stress and strain curves of an AZ31B sheet with very strong basal texture under cyclic loading along the RD (a), and the normalized hardening rate and elastic strain rate as functions of accumulated strain (b). The experimental data are taken from $\mathrm{Wu}$ et al. [40], and the values of the hardening parameters are from Guo et al. [39]. 


\section{texture $1 \quad$ texture 2 texture 3 texture 4}

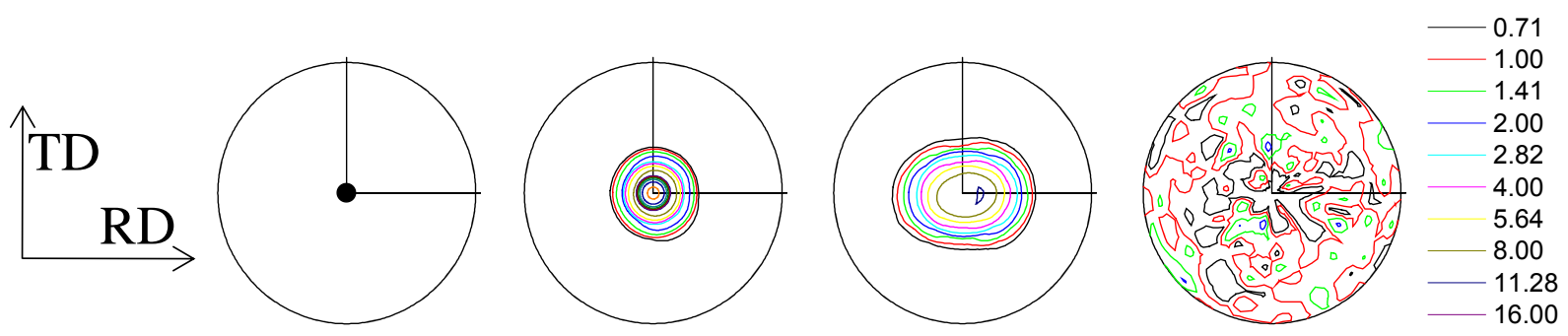

Figure 1. Initial textures presented in terms of the $\{0001\}$ pole figure. Textures $1-4$ are, from left to right, an ideal orientation with Euler angles $\left(30^{\circ}, 0^{\circ}, 0^{\circ}\right)$, a very strong basal texture of AZ31B from Wu et al. [49], a strong basal texture of AZ31B from Jain and Agnew [47], and a random texture.

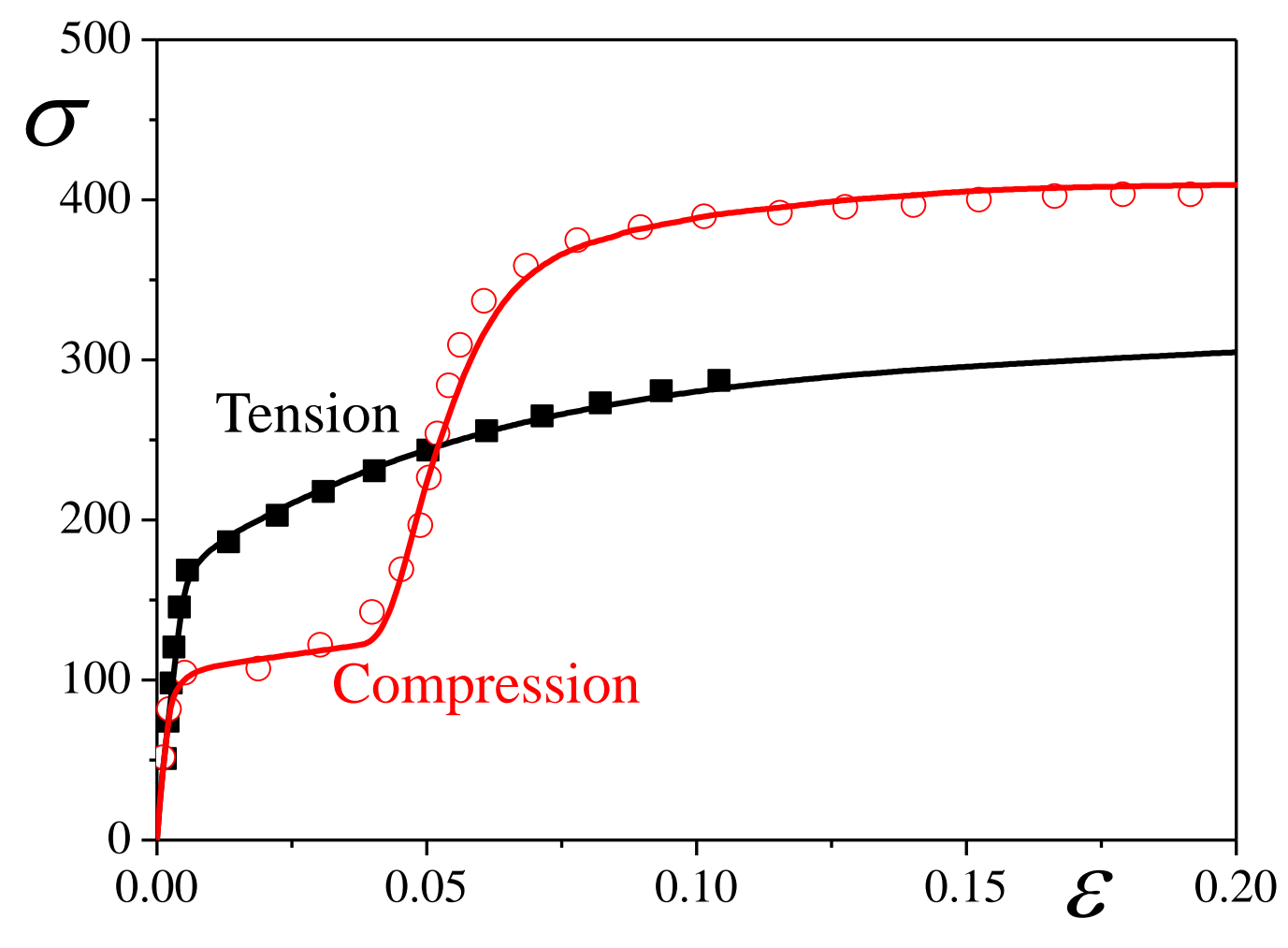

Figure 2. Measured (symbols) and calculated (solid lines) stress and strain curves under uniaxial compression and tension along the RD. The initial texture (texture 3 in Fig. 1) and experimental stress and strain data are from Jain and Agnew [47]. 


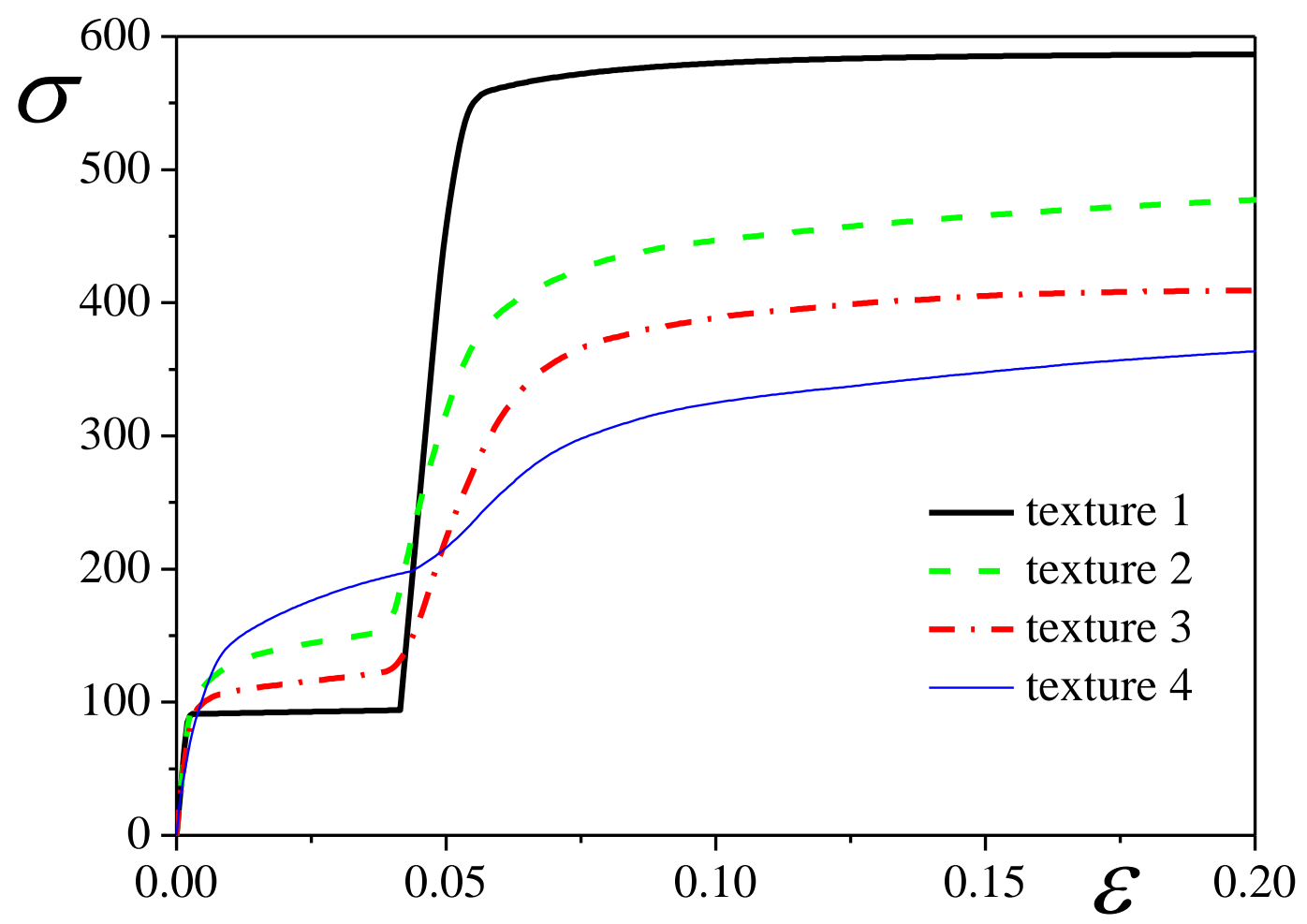

Figure 3. Calculated effect of initial texture on stress and strain curve under uniaxial compression along the RD. 

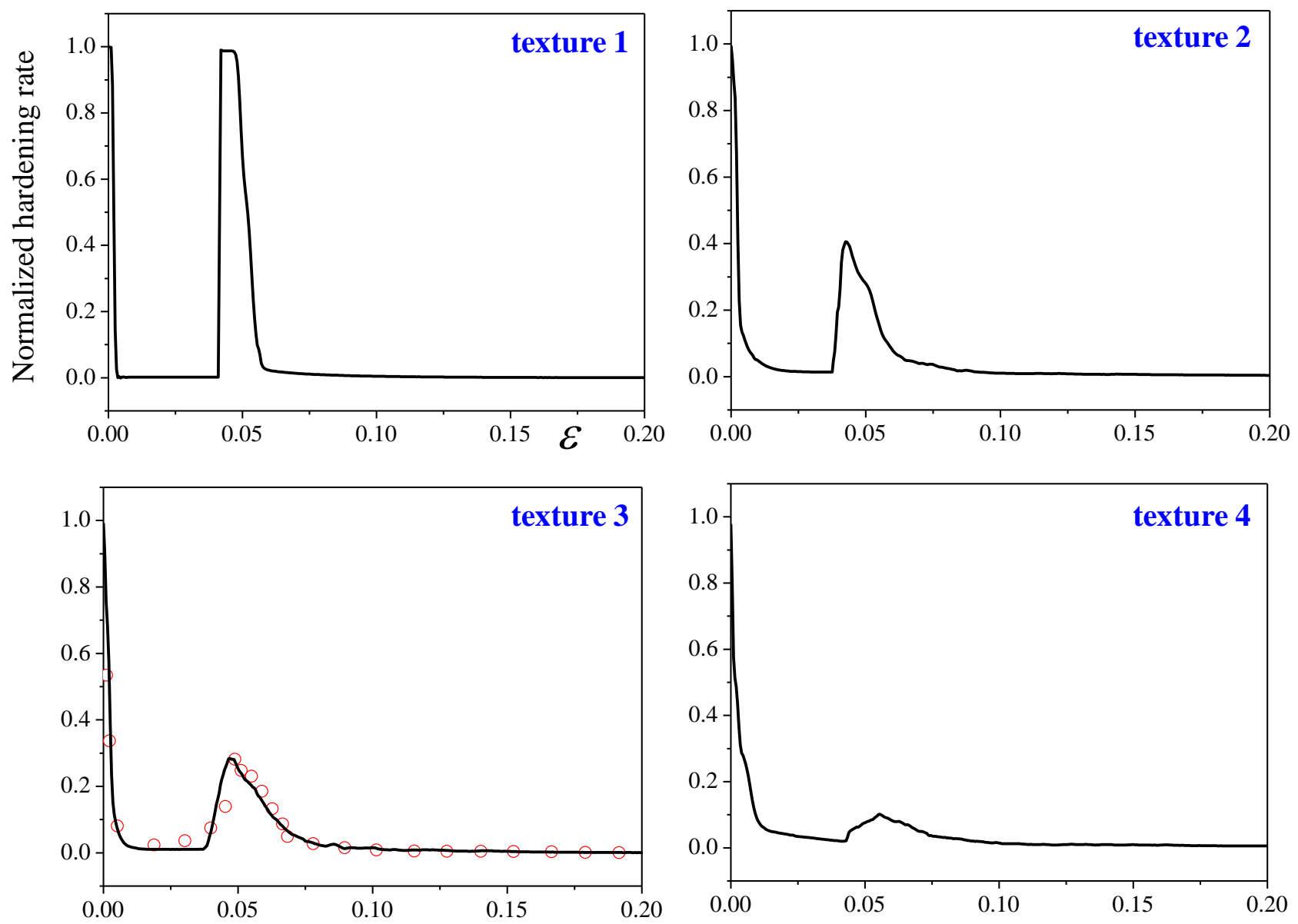

Figure 4. Calculated effect of initial texture on work hardening rate under uniaxial compression along the RD. The experimental data (open circles) for texture 3 are from Jain and Agnew [47]. 

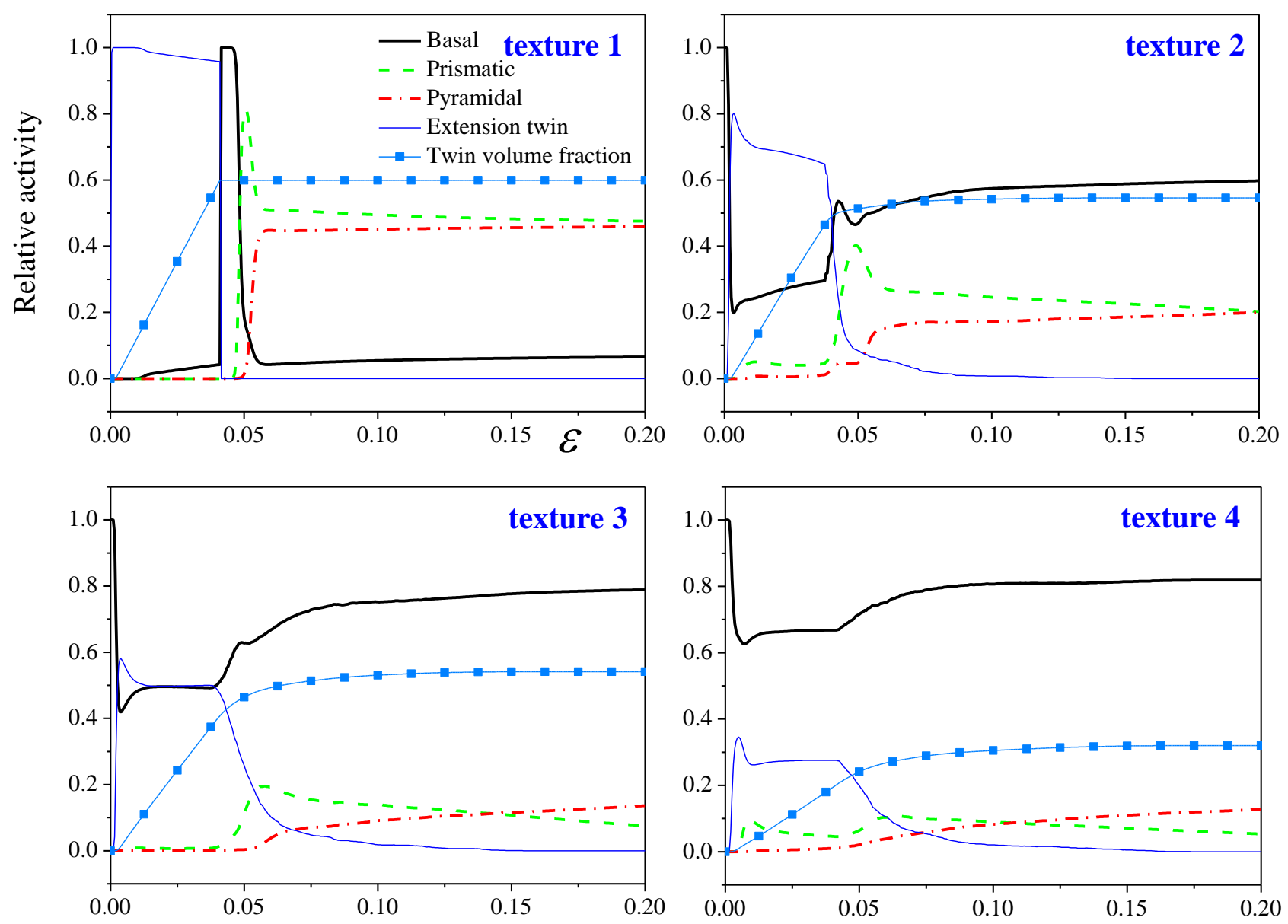

Figure 5. Calculated effect of initial texture on relative activities of the slip modes and tensile twinning under uniaxial compression along the RD. 

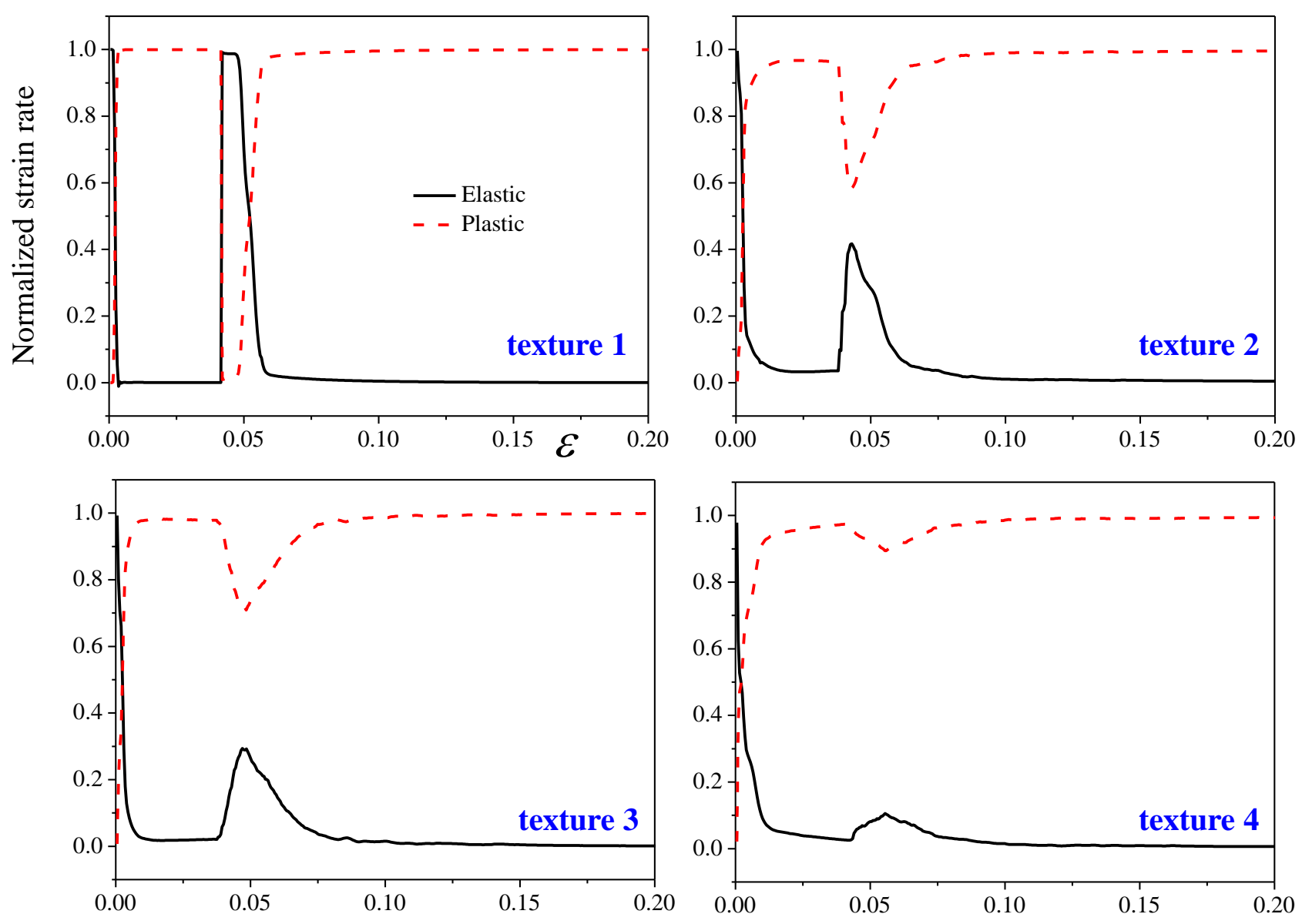

Figure 6. Calculated effect of initial texture on normalized plastic strain rate (dashed lines) and elastic strain rate (solid lines) under uniaxial compression along the RD. 

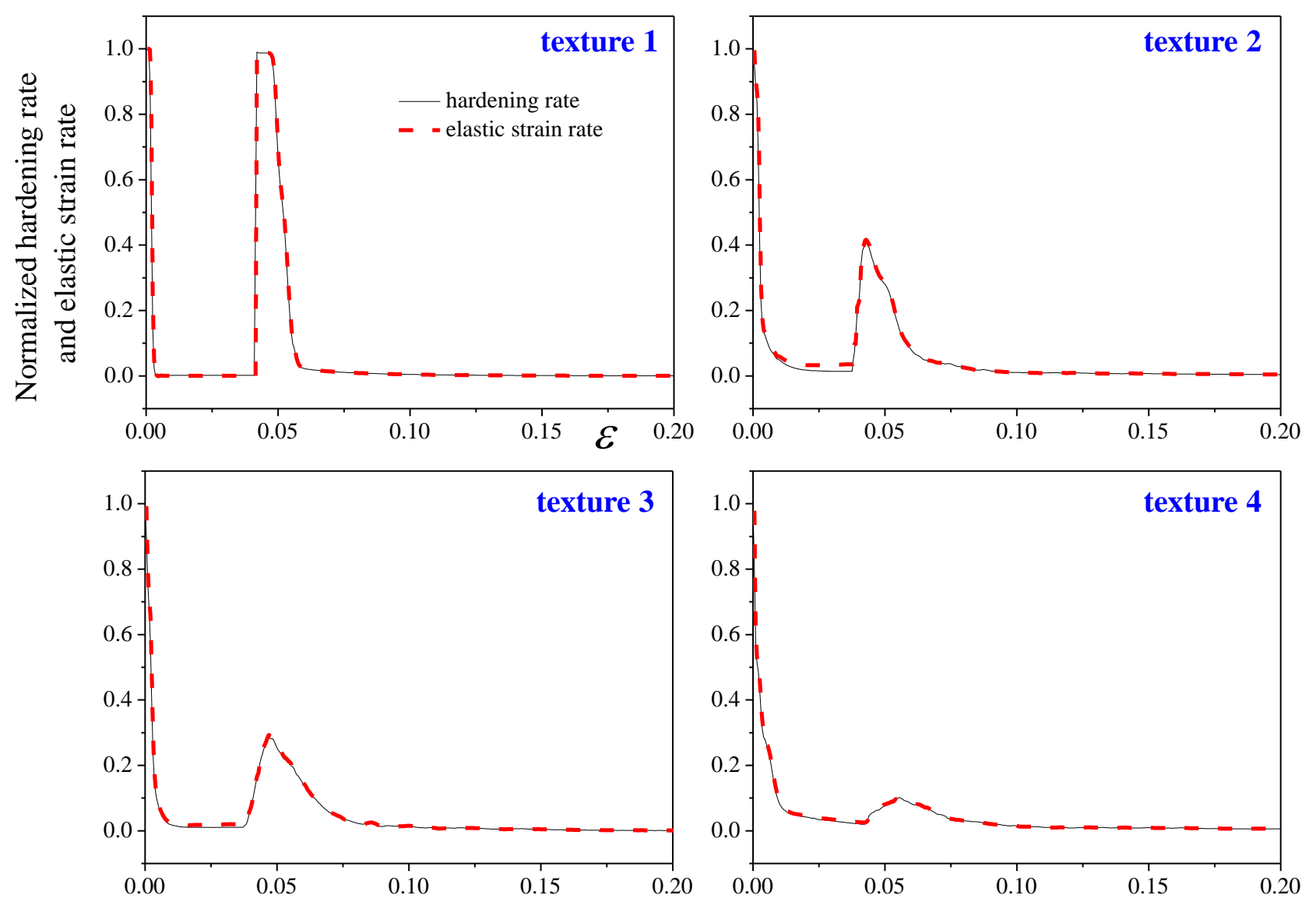

Figure 7. Calculated normalized hardening rate (thin black solid lines) and normalized elastic strain rate (thick red dashed lines) under uniaxial compression along the RD. 


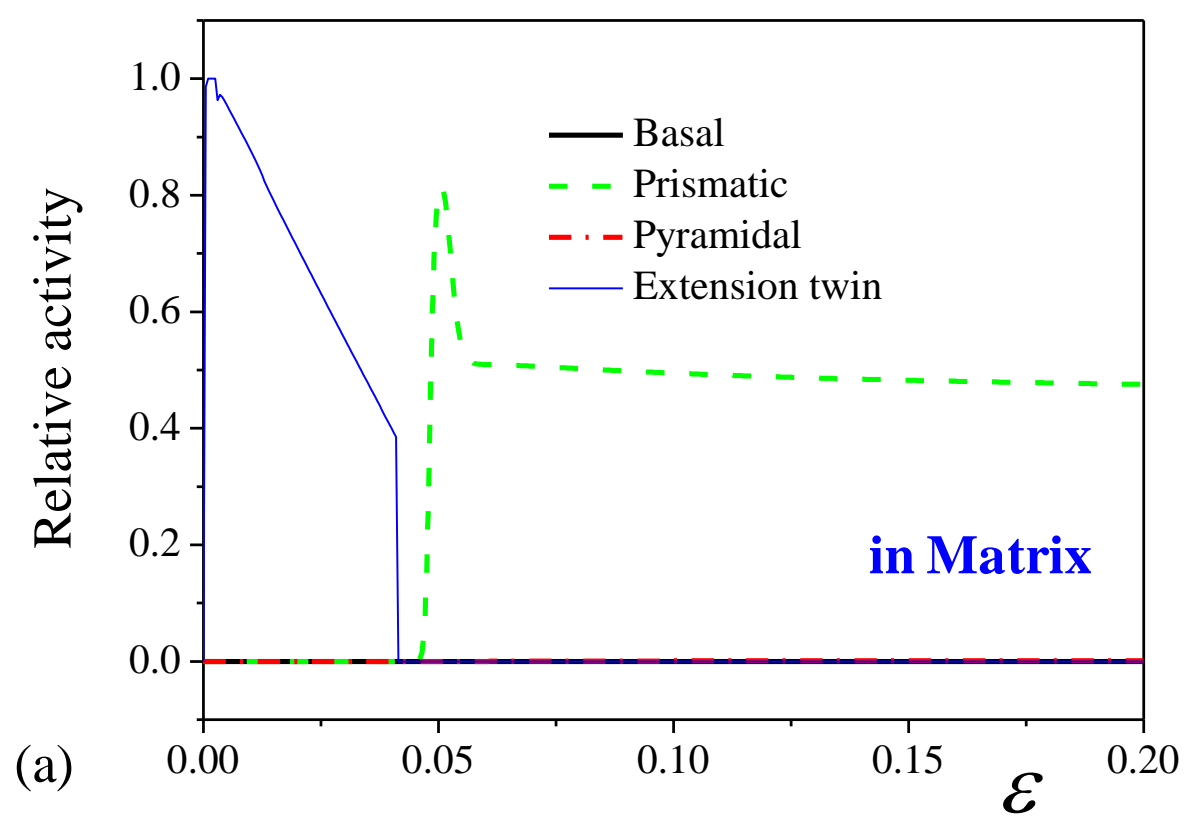

(a)

(b)

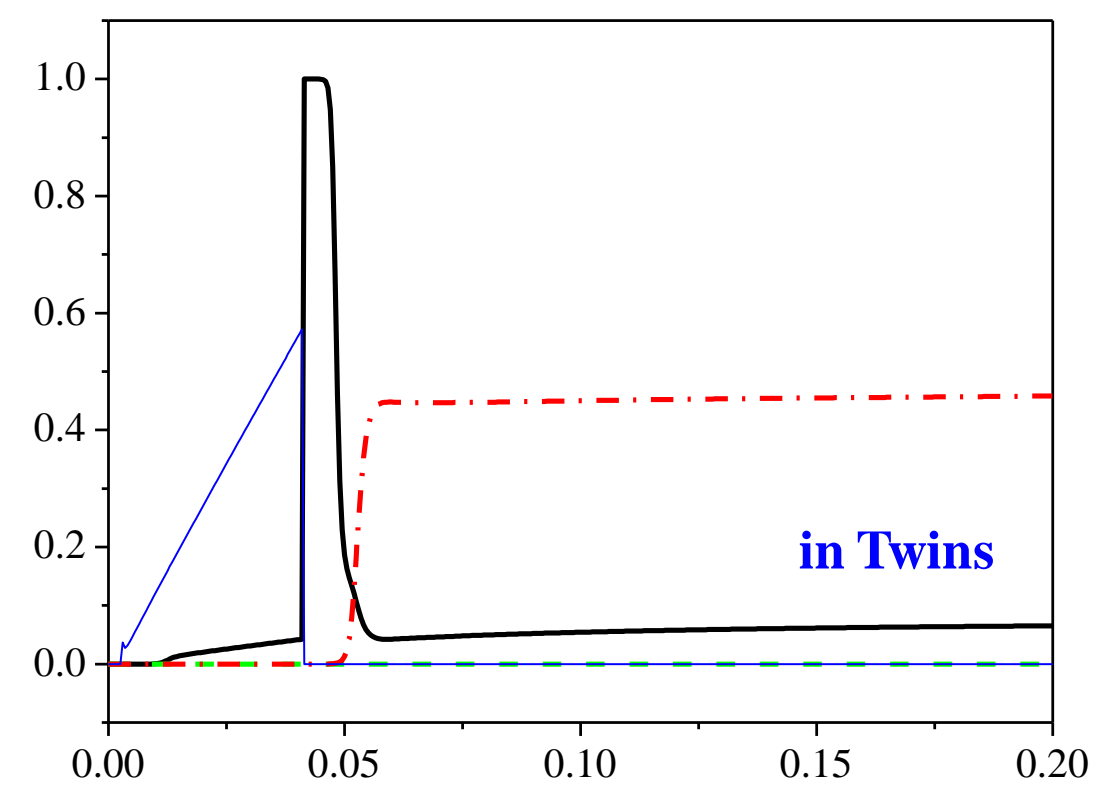

Figure 8. Calculated relative activities of the slip modes and tensile twinning in the matrix and twins for the ideal basal texture. Note that the total of the relative activities were presented in Figure 5. 

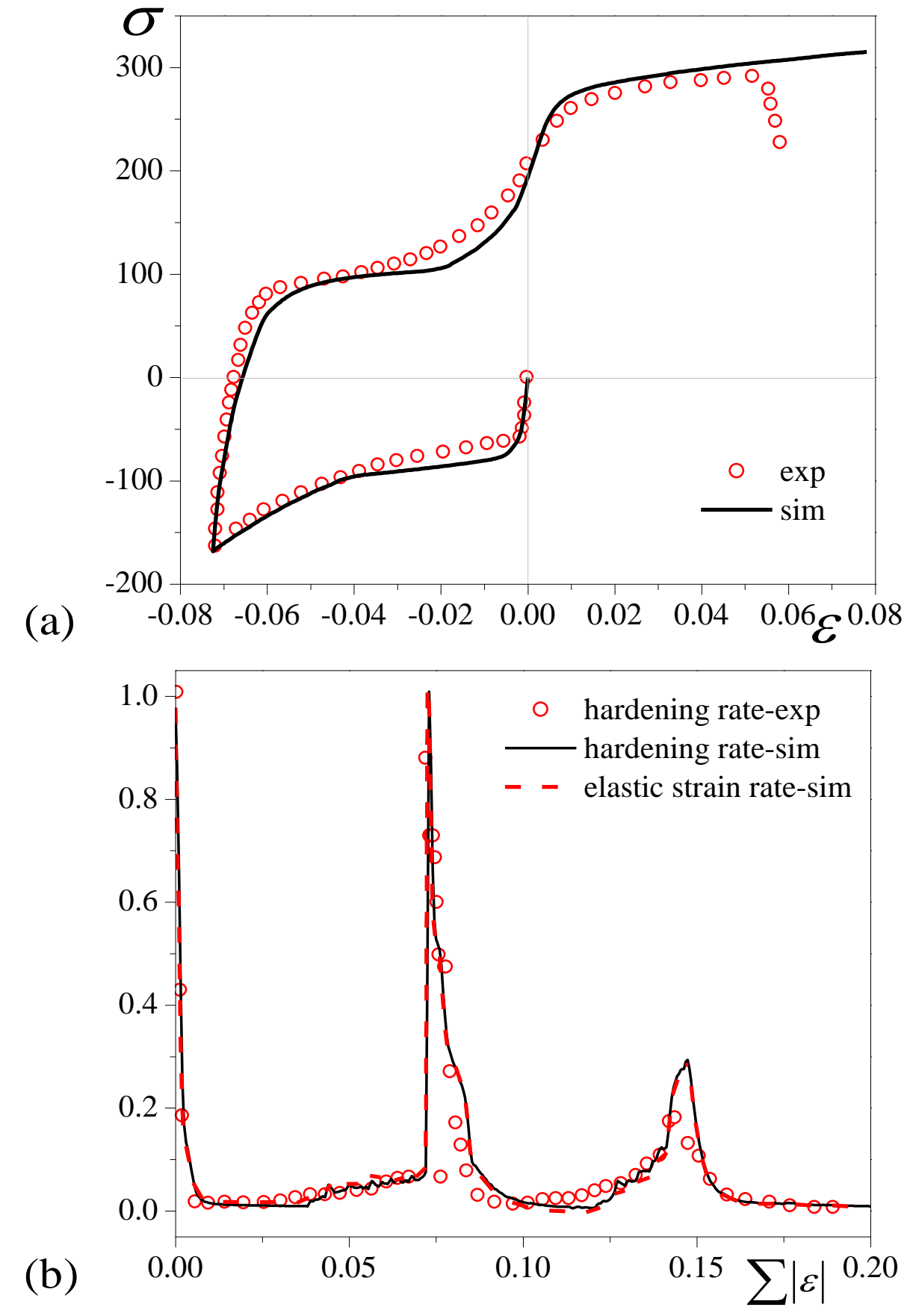

Figure 9. Measured and calculated stress and strain curves of an AZ31B sheet with very strong basal texture under cyclic loading along the RD (a), and the normalized hardening rate and elastic strain rate as functions of accumulated strain (b). The experimental data are taken from Wu et al. [40], and the values of the hardening parameters are from Guo et al. [39]. 
Table 1. List of values of the hardening parameters involved in the EVPSC-TDT model.

\begin{tabular}{cccccccc}
\hline Mode & $\boldsymbol{\tau}_{\mathbf{0}}(\mathrm{MPa})$ & $\boldsymbol{\tau}_{\mathbf{1}}(\mathrm{MPa})$ & $\boldsymbol{h}_{\mathbf{0}}(\mathrm{MPa})$ & $\boldsymbol{h}_{\mathbf{1}}(\mathrm{MPa})$ & $\boldsymbol{h}^{\alpha \beta}$ & $\boldsymbol{A}_{\mathbf{1}}$ & $\boldsymbol{A}_{\mathbf{2}}$ \\
\hline Basal & $\mathbf{1 0}$ & 1 & 500 & 0 & 4 & & \\
Prismatic & $\mathbf{7 5}$ & 50 & 450 & 0 & 4 & & \\
Pyramidal & $\mathbf{1 4 0}$ & 65 & 2500 & 0 & 2 & & \\
Extension twin & 42 & 0 & 0 & 0 & 4 & 0.60 & 0.0 \\
\hline
\end{tabular}



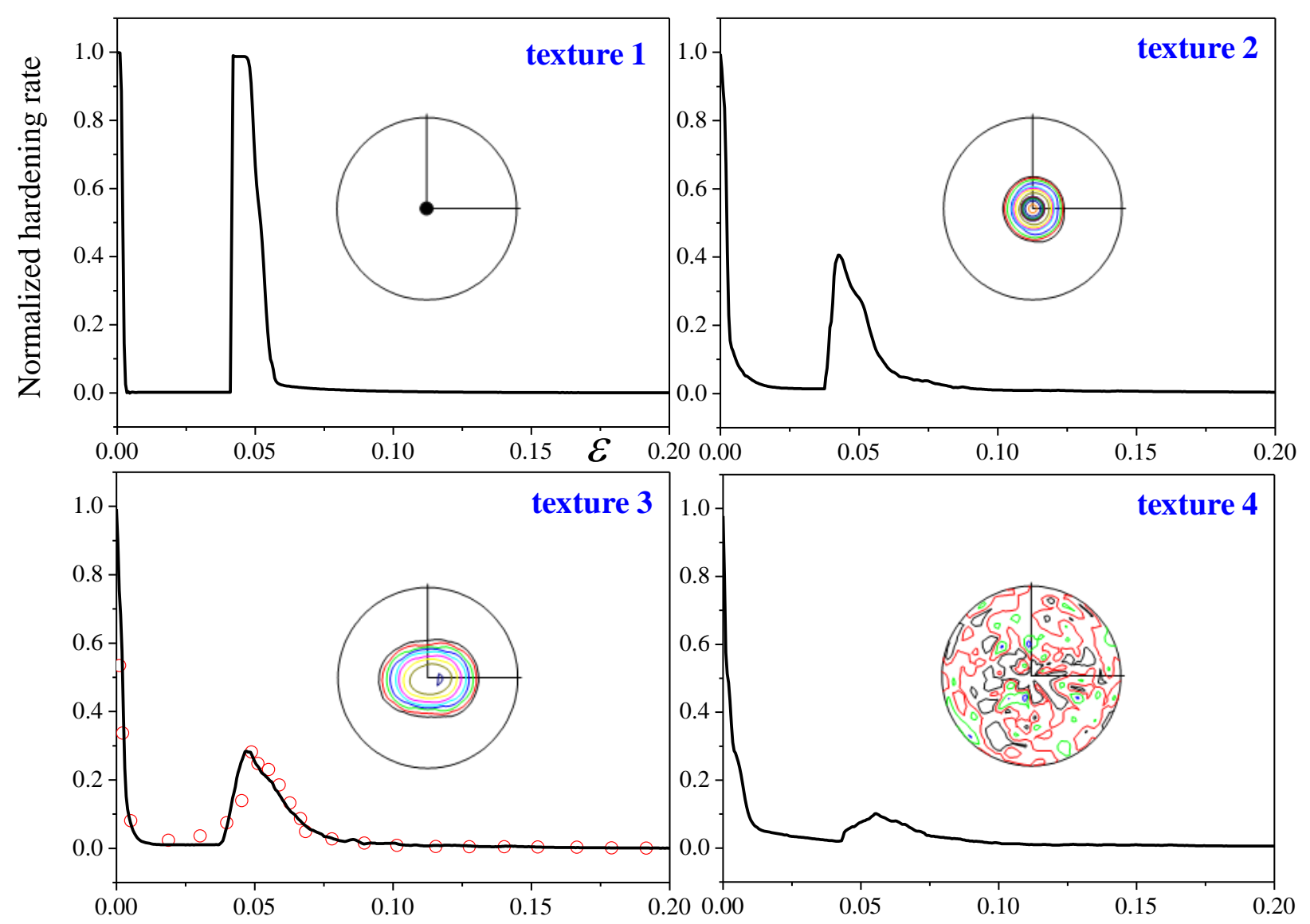